\title{
Nonlinear TE-polarized surface polaritons on graphene
}

\author{
Yuliy V. Bludov, ${ }^{1, *}$ Daria A. Smirnova, ${ }^{2}$ Yuri S. Kivshar, ${ }^{2}$ N. M. R. Peres, ${ }^{1}$ and Mikhail I. Vasilevskiy ${ }^{1}$ \\ ${ }^{1}$ Centro de Física and Departamento de Física, Universidade do Minho, Campus de Gualtar, Braga 4710-057, Portugal \\ ${ }^{2}$ Nonlinear Physics Center, Research School of Physics and Engineering, Australian National University, Canberra ACT 0200, Australia
}

(Received 5 August 2013; revised manuscript received 1 December 2013; published 7 January 2014)

\begin{abstract}
We analyze the propagation of electromagnetic waves along the surface of a nonlinear dielectric medium covered by a graphene layer. We reveal that this system can support and stabilize nonlinear transverse electric (TE) plasmon polaritons. We demonstrate that these nonlinear TE modes have a subwavelength localization in the direction perpendicular to the surface, with the intensity much higher than that of an incident wave which excites the polariton.
\end{abstract}

DOI: 10.1103/PhysRevB.89.035406

PACS number(s): 78.67.Wj, 42.65.Wi, 73.25.+i

\section{INTRODUCTION}

Nonlinear plasmonics is still a young but fast growing research field. ${ }^{1}$ It encompasses both a nonlinear response of an active medium — such as metal — and that of a surrounding dielectric medium. Nonlinear response of plasmonic systems has been observed both in metal films and in metallic nanostructures. ${ }^{1}$ In the present context, from many nonlinear effects allowed by nonlinear optics, second-harmonic generation and self-action Kerr effect have been the most studied. Kerr effect refers to the modification of the refractive index of a system by the electric field. In this case, the nonlinear susceptibility depends on the intensity of the electric field.

Plasmonics in graphene ${ }^{2}$ is a recent and intense field of research, impelled by many theoretical proposals ${ }^{3,4}$ and experimental results. ${ }^{5}$ In particular, it has been shown that graphene supports $p$-polarized surface plasmon polaritons, or transverse magnetic (TM) surface waves, ${ }^{4}$ with subwavelength localization in the direction perpendicular to the surface. Contrary to an ordinary metal, it has been shown that graphene also supports TE-type electromagnetic surface waves ${ }^{6,7}$ in a well defined and narrow frequency window. This type of surface waves exist in graphene as a consequence that the imaginary part of its interband optical conductivity may become negative. The existence of such a type of wave was invoked to explain, e.g., the broadband polarizing effect of graphene ${ }^{8}$ (see also Ref. 9). The TE waves can also be important in multilayer structures. ${ }^{9,10}$ Unfortunately, the degree of localization of the TE-type surface plasmon polaritons around the graphene layer is very weak, with the electromagnetic field behaving almost as free radiation.

In the realm of linear optics there is no hope that the degree of localization of the TE-type electromagnetic wave can be enhanced. ${ }^{11}$ Fortunately, nonlinear optics rescue us from this limitation. The idea is relatively simple. For a TE wave, the solutions of Maxwell's equation in the Kerr regime support localized fields, described by hyperbolic functions. Then, a simple experimental setup can be envisioned: A graphene sheet is cladded by two dielectrics, one being linear (for example, air) and the other nonlinear (for example, some special type of polymer). Electromagnetic radiation is let to impinge from the linear dielectric onto graphene and a surface wave is excited (e.g., with the help of a microfabricated grating). Due to the presence of the nonlinear dielectric underneath, the field is localized in the vicinity of the interface. Furthermore, the enhancement of the electric field associated with the formation of the surface wave further enhances the nonlinear effect. Moreover, as we will see below, the presence of the nonlinear dielectric also frees us from the narrow frequency window predicted by the linear theory for the existence of TE waves. Indeed, it is shown that this type of wave can exist even if the imaginary part of the conductivity is positive. The main results, presented in this paper, are

(1) Subwavelength localization of TE waves around the graphene-covered surface or interface;

(2) enlarged energy window for the existence of TE waves;

(3) strong enhancement of the localized field relatively to its value in free space; and

(4) stability of the TE waves, which have a soliton character.

All these aspects are promoted by the presence of the nonlinear dielectric in the vicinity of graphene. In contrast with Ref. 12, where the subwavelength localization of TE waves was caused by intrinsic nonlinearity of graphene at optical frequencies, here we demonstrate the existence of spatial solitons at $\mathrm{THz}$ to far infrared frequencies, originated from substrate nonlinearity and supported by plasma oscillations in graphene layer. In what follows we will see how these results appear from a simple formulation of the nonlinear problem.

The paper is organized as follows. In Sec. II we discuss the geometry of our problem and introduce the basic equations. In Sec. III the dispersion properties of nonlinear surface polaritons are discussed, while the stability results are summarized in Sec. IV. Section V concludes the paper.

\section{MODEL}

We consider a graphene sheet covering a flat interface (at $z=0$ ) between two semi-infinite dielectric media (see Fig. 1). The upper dielectric has a linear dielectric permittivity $\varepsilon_{2}>0$, and the nonlinear substrate has a dielectric permittivity $\varepsilon_{1}^{\mathrm{NL}}=\varepsilon_{1}+\chi^{(3)}|E|^{2}$; then we are exploiting here the Kerr effect (the parameter $\chi^{(3)}>0$ is the nonlinear coefficient of the self-focusing medium). We assume that the substrate and the capping dielectric occupy the half-spaces $z>0$ and $z<0$, respectively. Moreover, we suppose that the incident electromagnetic radiation, in the form of TE waves, is uniform along the direction $y$, with the field vectors given by $\vec{E}=$ $\left\{0, E_{y}, 0\right\}$ and $\vec{H}=\left\{H_{x}, 0, H_{z}\right\}$. The temporal dependence of 


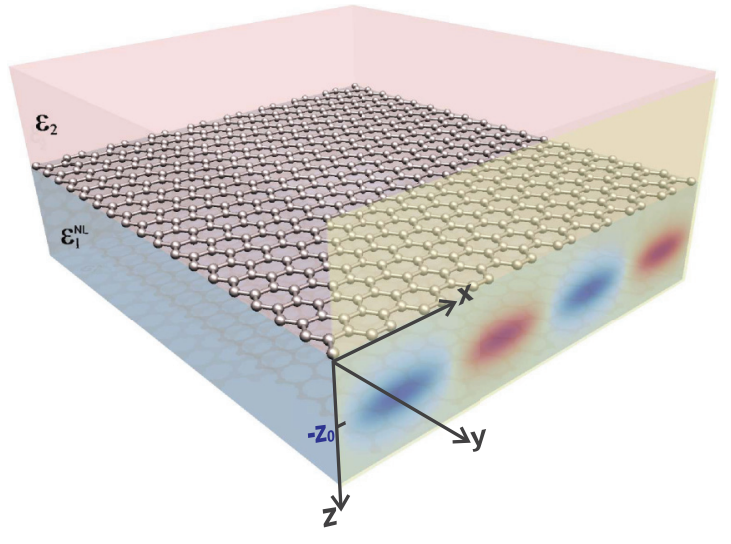

FIG. 1. (Color online) Schematics of a graphene layer separating the upper linear and lower nonlinear dielectric media with the dielectric permittivities $\varepsilon_{2}$ and $\varepsilon_{1}^{\mathrm{NL}}$, respectively, shown with a spatial profile of the nonlinear surface polariton.

the electromagnetic field has the standard form $\sim \exp (-i \omega t)$, where $\omega$ is the frequency.

Maxwell's equations for a TE wave can be written in the form

$$
\begin{aligned}
& \frac{\partial H_{x}^{(j)}}{\partial z}-\frac{\partial H_{z}^{(j)}}{\partial x}=-i \omega \varepsilon_{0}\left[\varepsilon_{j}+\delta_{j, 1} \chi^{(3)}\left|E_{y}^{(j)}\right|^{2}\right] E_{y}^{(j)} \\
& \frac{\partial E_{y}^{(j)}}{\partial z}=-i \omega \mu_{0} H_{x}^{(j)}, \quad \frac{\partial E_{y}^{(j)}}{\partial x}=i \omega \mu_{0} H_{z}^{(j)} ; \quad j=1,2 .
\end{aligned}
$$

Introducing the dimensionless and slowly varying amplitudes of the electromagnetic field $\mathcal{E}_{y}^{(j)}(x, z)=\left(\chi^{(3)} / 2\right)^{1 / 2} E_{y}^{(j)}$ $\exp \left(-i k_{x} x\right)$ and $\mathcal{H}_{x, z}^{(j)}(x, z)=\left(\chi^{(3)} / 2\right)^{1 / 2} c \mu_{0} H_{x, z}^{(j)} \exp \left(-i k_{x} x\right)$, it follows from the above equations that

$$
\begin{aligned}
& 2 i k_{x} \frac{\partial \mathcal{E}_{y}^{(j)}}{\partial x}+\frac{\partial^{2} \mathcal{E}_{y}^{(j)}}{\partial z^{2}}-k_{x}^{2} \mathcal{E}_{y}^{(j)} \\
& \quad+\left(\frac{\omega}{c}\right)^{2}\left[\varepsilon_{j}+2 \delta_{j, 1}\left|\mathcal{E}_{y}^{(j)}\right|^{2}\right] \mathcal{E}_{y}^{(j)}=0,
\end{aligned}
$$

where $k_{x}$ is the in-plane component of the wave vector and $\delta_{j, m}$ is the Kronecker symbol.

\section{NONLINEAR SURFACE MODES}

In the nonlinear medium $(j=1)$, the stationary ( $x$-independent) solution of wave equation (1) can be represented in the form of localized modes. Indeed, the solution has the simple form of bright soliton, ${ }^{13}$

$$
\begin{gathered}
\mathcal{E}_{y}^{(1)}(z)=\frac{c p_{1}}{\omega} \frac{1}{\cosh \left[p_{1}\left(z+z_{0}\right)\right]}, \\
\mathcal{H}_{x}^{(1)}(z)=\frac{c^{2} p_{1}^{2}}{i \omega^{2}} \frac{\sinh \left[p_{1}\left(z+z_{0}\right)\right]}{\cosh ^{2}\left[p_{1}\left(z+z_{0}\right)\right]} .
\end{gathered}
$$

It is clear that the wave is localized around $z=-z_{0}$; this type of solution is sometimes called a spatial soliton. The parameter $z_{0}$ can be either positive or negative. We are most interested in the case where the TE wave is localized in the nonlinear medium, for which $z_{0}<0$.
On the other hand, in the linear medium $(j=2)$ the solution has the standard form:

$$
\begin{gathered}
\mathcal{E}_{y}^{(2)}(z)=\mathcal{E}_{y}(0) \exp \left(p_{2} z\right), \\
\mathcal{H}_{x}^{(2)}(z)=-\frac{c p_{2}}{i \omega} \mathcal{E}_{y}(0) \exp \left(p_{2} z\right) .
\end{gathered}
$$

In the above we have introduced the parameter $p_{j}^{2}=k_{x}^{2}-$ $(\omega / c)^{2} \varepsilon_{j}$. The electric field at the interface $\mathcal{E}_{y}(0)$, and the dispersion relation for the TE waves are determined from the boundary conditions. These are $\mathcal{E}_{y}^{(2)}(0)=\mathcal{E}_{y}^{(1)}(0)$ and $\mathcal{H}_{x}^{(1)}(0)-\mathcal{H}_{x}^{(2)}(0)=c \mu_{0} \sigma(\omega) \mathcal{E}_{y}^{(2)}(0)$, from which we have

$$
\begin{gathered}
\frac{c p_{1}}{\omega} \frac{1}{\cosh \left[p_{1} z_{0}\right]}=\mathcal{E}_{y}(0), \\
\frac{c^{2} p_{1}^{2}}{i \omega^{2}} \frac{\sinh \left[p_{1} z_{0}\right]}{\cosh ^{2}\left[p_{1} z_{0}\right]}+\frac{c p_{2}}{i \omega} \mathcal{E}_{y}(0)=c \mu_{0} \sigma(\omega) \mathcal{E}_{y}(0) .
\end{gathered}
$$

Combining these two equations we obtain the dispersion relation $p_{1} \tanh \left(p_{1} z_{0}\right)+p_{2}=i \omega \mu_{0} \sigma(\omega)$, or, equivalently,

$$
s\left[p_{1}^{2}-\left(\frac{\omega}{c}\right)^{2}\left|\mathcal{E}_{y}(0)\right|^{2}\right]^{1 / 2}+p_{2}=i \omega \mu_{0} \sigma(\omega),
$$

where $s= \pm 1$ stands for the sign of the parameter $z_{0}$. We note that the dispersion relation of the nonlinear surface polaritons is determined by the graphene conductivity $\sigma(\omega)$, which has been given, for example, in Ref. 3 and depends on the Fermi energy $\mu$. The general trend of the optical conductivity of graphene is as follows: In the low-frequency range, the Drude term exceeds significantly the interband contribution, for both the real and imaginary parts, while in the high-frequency range (that is, close to twice the Fermi energy) the latter contribution dominates. Moreover, in the vicinity of the frequency $\omega=2 \mu$ the real part of the graphene conductivity increases drastically and achieves the universal conductivity value $\sigma_{0}=\pi e^{2} /(2 h)$ ( $h$ is the Planck constant), whereas the imaginary part, which is negative, attains its minimum value. Also, there exists a special frequency $\omega_{*}$, where the imaginary part of the conductivity $\sigma^{\prime \prime}=\operatorname{Im}[\sigma(\omega)]$ vanishes, i.e., $\sigma^{\prime \prime}\left(\omega_{*}\right)=0$.

Without the graphene layer, the TE wave could exist only in the case $s=-1$ for a single value of the electric field amplitude at the interface, ${ }^{14}$ determined by $\left|\mathcal{E}_{y}(0)\right|^{2}=\varepsilon_{2}-\varepsilon_{1} \equiv-\Delta_{0}$ (for $\Delta_{0}<0$ ), and for any wave vector $k_{x} \geqslant \omega \varepsilon_{2}^{1 / 2} / c$. On the contrary, and most importantly, in the case where a graphene layer is present, the existence range for the TE wave becomes significantly wider. Indeed, Eq. (8) can be rewritten in terms of $\Delta=\varepsilon_{1}-\varepsilon_{2}+\left|\mathcal{E}_{y}(0)\right|^{2} \equiv \Delta_{0}+\left|\mathcal{E}_{y}(0)\right|^{2} \geqslant \Delta_{0}$, as

$$
s\left[p_{2}^{2}-\left(\frac{\omega}{c}\right)^{2} \Delta\right]^{1 / 2}+p_{2}=-\omega \mu_{0} \sigma^{\prime \prime},
$$

where the real part of the graphene conductivity $\sigma^{\prime}=$ $\operatorname{Re}[\sigma(\omega)] \equiv 0$ has been neglected for clarity (this corresponds to the limit of a dispersive dielectric). The above equation can be solved for $p_{2}$ and we obtain

$$
p_{2}=-\frac{\Delta+c^{2} \mu_{0}^{2} \sigma^{\prime \prime 2}}{2 c^{2} \mu_{0} \omega^{-1} \sigma^{\prime \prime}},
$$


which links $\omega, k_{x}$, and $\Delta$. One can consider $\omega$ and $\Delta$ as independently controlled parameters determined by the excitation conditions (in particular, $\Delta$ can be adjusted via the intensity of the incident wave) and, contrary to the case without graphene, here $k_{x}$ is uniquely determined by $\omega$ and $\Delta$.

Equation (10) allows for a simple qualitative analysis. The case $\sigma^{\prime \prime}>0$ is the simplest one. Here the positiveness of $p_{2}$ can be achieved only when the numerator of Eq. (10) is negative. Thus, the surface waves exist when

$$
\Delta<-c^{2} \mu_{0}^{2} \sigma^{\prime \prime 2}
$$

and $s=-1$ [last condition stems from the requirement that the left-hand side of Eq. (9) should be negative]. We stress that this result is in contrast with the linear regime, ${ }^{6}$ where only for
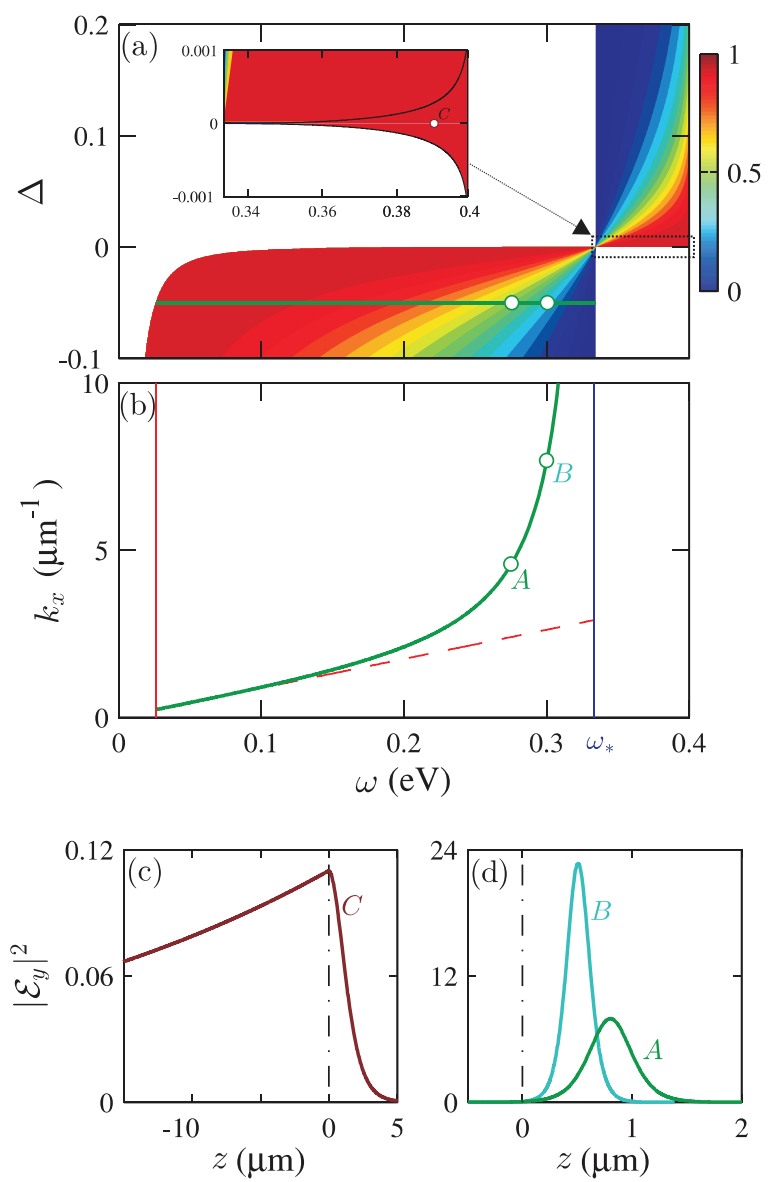

FIG. 2. (Color online) (a) Surface polariton dimensionless phase velocity $\omega \sqrt{\varepsilon_{2}} / c k_{x}$ versus frequency and parameter $\Delta$. Inset in (a) depicts zoom in the vicinity of $\Delta=0$. (b) Dispersion relation $k_{x}(\omega)$ (solid line) for fixed $\Delta=-0.05$ [extracted from (a) along the respective horizontal line] and the light line in the linear dielectric $k_{x}=(\omega / c) \sqrt{\varepsilon_{2}}$ (dashed red line). (c) and (d) Spatial profiles $\left|\mathcal{E}_{y}(z)\right|^{2}$ of surface polaritons for $\Delta=0, \omega=0.39 \mathrm{eV}$ [curve $\mathrm{C}$ in (c)] and $\Delta=-0.05, \omega=0.275 \mathrm{eV}$, or $\omega=0.3 \mathrm{eV}$ [curves $\mathrm{A}$ and $\mathrm{B}$ in (d), correspondingly]. The curves A-C correspond to the respective points in (a) and (b). The interface between the linear and nonlinear dielectrics is depicted by a vertical dash-dotted black line. In all panels the dielectric permittivities of the nonlinear and linear media are $\varepsilon_{1}=2.89, \varepsilon_{2}=3$, while the relaxation rate and the chemical potential of graphene are $\Gamma=0$ and $\mu=0.2 \mathrm{eV}$. $\sigma^{\prime \prime}<0$ TE waves can propagate on a graphene-covered interface. Notice that the upper limit $\Delta=-c^{2} \mu_{0}^{2} \sigma^{\prime \prime 2}$ corresponds to the case $p_{2}=0$, which implies fully delocalized wave. On the other hand, for the case $\sigma^{\prime \prime}<0$ the surface waves exist when numerator of Eq. (10) is positive. Namely, they exist in the domains

$$
-c^{2} \mu_{0}^{2} \sigma^{\prime \prime 2}<\Delta<c^{2} \mu_{0}^{2} \sigma^{\prime \prime 2}
$$

for $s=1$ (this is the less interesting case) and

$$
\Delta>c^{2} \mu_{0}^{2} \sigma^{\prime \prime 2},
$$

for $s=-1$. In conclusion, the existence domains for $s=-1$ are very wide. We notice that in the linear limit the nonlinear surface polariton with $s=+1$ transforms into a TE-polarized surface polariton described in Ref. 6. At the same time, the
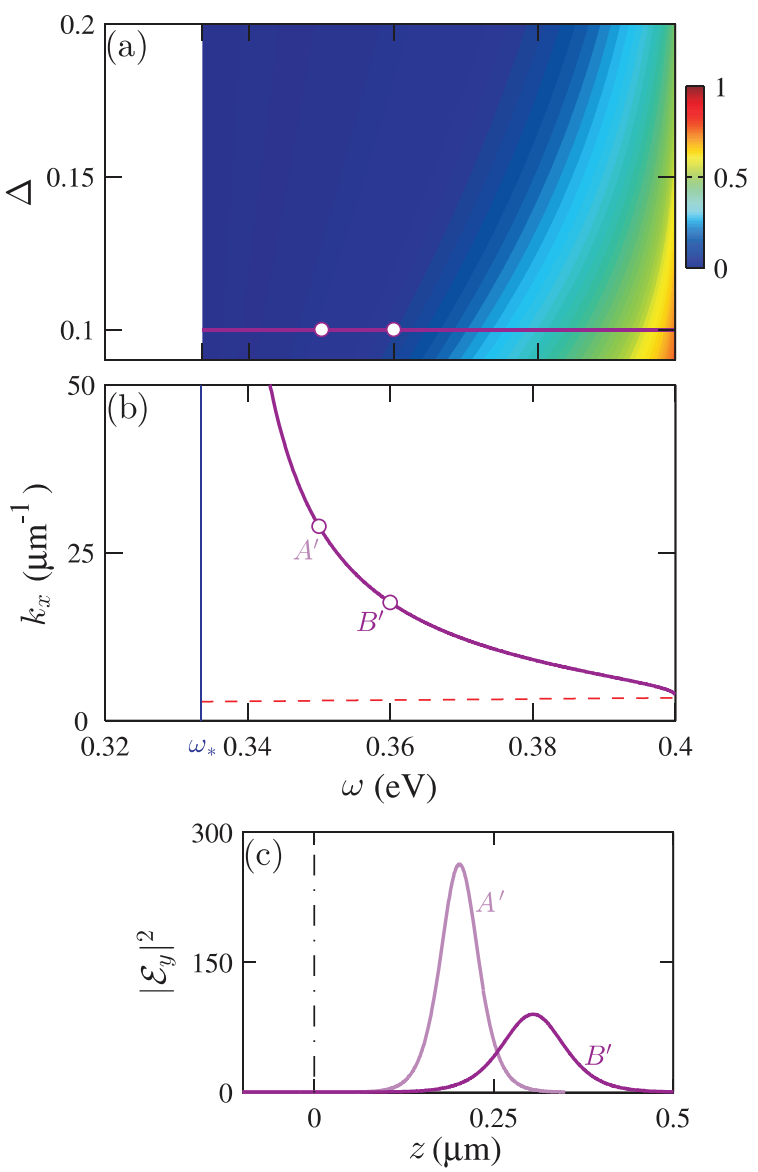

FIG. 3. (Color online) (a) Surface polariton dimensionless phase velocity $\omega \sqrt{\varepsilon_{2}} / c k_{x}$ versus frequency and parameter $\Delta$. (b) Dispersion relation $k_{x}(\omega)$ (solid line) corresponding to the horizontal line $\Delta=$ 0.1 in (a) and the light line in the linear dielectric $k_{x}=(\omega / c) \sqrt{\varepsilon_{2}}$ (dashed red line). (c) Spatial profiles $\left|\mathcal{E}_{y}(z)\right|^{2}$ of surface polaritons for the parameters: $\Delta=0.1, \omega=0.35 \mathrm{eV}$, or $\omega=0.36 \mathrm{eV}$ (curves $\mathrm{A}^{\prime}$ and $\mathrm{B}^{\prime}$, correspondingly). Curves $\mathrm{A}^{\prime}$ and $\mathrm{B}^{\prime}$ correspond to the respective points in (a) and (b). The interface between the linear and nonlinear dielectrics is depicted by a vertical dash-dotted black line. In all panels the dielectric permittivities of the nonlinear and linear media are $\varepsilon_{1}=2.89, \varepsilon_{2}=2.8$, while the relaxation rate and the chemical potential of graphene are $\Gamma=0$ and $\mu=0.2 \mathrm{eV}$. 
nonlinear surface polariton with $s=-1$ is a purely nonlinear object; it does not have its linear counterpart.

The existence domains are depicted in Figs. 2(a) and 3(a) for the cases $\varepsilon_{1}<\varepsilon_{2}$ and $\varepsilon_{1}>\varepsilon_{2}$, respectively. In both plots, the white color represents domains where the nonlinear surface polaritons cannot exist, while the bottom limits in these plots correspond to the limiting value $\Delta=\Delta_{0}$.

For $\varepsilon_{1}<\varepsilon_{2}$ [see Fig. 2(a)] there are three existence domains: (i) the finite domain at frequency range $\omega<\omega_{*}$, characterized by positive $\sigma^{\prime \prime}>0$, negative $s=-1$, and restricted by the curves (11), $\Delta=\Delta_{0}$, and $\omega=\omega_{*}$; (ii) the infinite domain at frequency range $\omega>\omega_{*}$, characterized by negative $\sigma^{\prime \prime}<0$, positive $s=1$, and restricted by the curve (12) [see the inset in Fig. 2(a)]; and (iii) the infinite domain at frequency range $\omega>\omega_{*}$, characterized by negative $\sigma^{\prime \prime}<0$, negative $s=-1$, and restricted by the curves (13) and $\omega=\omega_{*}$. The last two regions are semi-infinite in frequency and $\Delta$.

For $\varepsilon_{1}>\varepsilon_{2}$ [see Fig. 3(a)] there is only one infinite domain at frequency range $\omega>\omega_{*}$ (although the $s=1$ domain is also present here, for these particular parameters it is just a narrow strip in the vicinity of the frequency $\omega=2 \mu$ ), characterized by negative $\sigma^{\prime \prime}<0$, negative $s=-1$, and restricted by the curves $\Delta=\Delta_{0}, \omega=\omega_{*}$, and Eq. (12).

The intensity scale in Figs. 2(a) and 3(a) corresponds to the phase velocity $\omega / k_{x}$ (in units of the velocity of light in the linear dielectric $\left.c / \sqrt{\varepsilon_{2}}\right)$. The phase velocity attains its minimum value in the vicinity of the frequency $\omega=\omega_{*}$ (where $\sigma^{\prime \prime}=0$ ) and decreases with an increase of $|\Delta|$. We notice that this behavior is different from the case of TM-polarized nonlinear surface polaritons, ${ }^{15}$ where the minimum of the phase velocity was achieved for all frequencies close to a certain value of $\Delta$. In Figs. 2(b) and 3(b) this behavior is seen in more detail, for the dispersion curves $k_{x}(\omega)$ obtained from Figs. 2(a) and 3(a) along the respective horizontal lines. From Figs. 2(b) and 3(b) it is seen that far from the frequency $\omega=\omega_{*}$ the wave vector $k_{x}$ is close to that in the linear dielectric, while in the vicinity of $\omega=\omega_{*}$ the wave vector $k_{x}$ significantly exceeds its counterpart in the linear medium.

A very important piece of information is the spatial profile of the electric field $\left|\mathcal{E}_{y}(z)\right|^{2}$. It is depicted in Figs. 2(c), 2(d), and 3(c). Here, in the case $s=1$ [see Fig. 2(c)] surface polariton profile resembles its linear counterpart- the electric field maximum is at the interface between linear and nonlinear media and the wave is poorly localized. At the same time, due to the nonlinearity of the substrate, the mode localization is much stronger for $z>0$ than in the linear dielectric. For the case $s=-1$ [Figs. 2(d) and 3(c)] the maximum of the spatial soliton is situated inside the nonlinear dielectric, and the increase of $k_{x}$ shifts it closer to the interface (smaller $\left|z_{0}\right|$ ) and is accompanied by an increase of the soliton amplitude and a decrease of its width (compare curves A and B, as well as curves $\mathrm{A}^{\prime}$ and $\mathrm{B}^{\prime}$ ). These are two central results of this work: The TE-type surface wave shows subwavelength localization and the intensity of the field is quite high. Such behavior is not found in the linear theory.

One of the advantages of graphene in electronics is the possibility to tune its conductivity by electrostatic gating. So, a natural question is how sensitive is the phase velocity of the nonlinear surface polariton with respect to the variation of the graphene chemical potential? We have found that for fixed values of the parameter $\Delta$ and frequency $\omega$, an increase of the chemical potential $\mu$ results in an increase (when $\varepsilon_{1}<\varepsilon_{2}$ ), or decrease (when $\varepsilon_{1}>\varepsilon_{2}$ ) of the phase velocity. Moreover, varying the chemical potential, the phase velocity can be varied
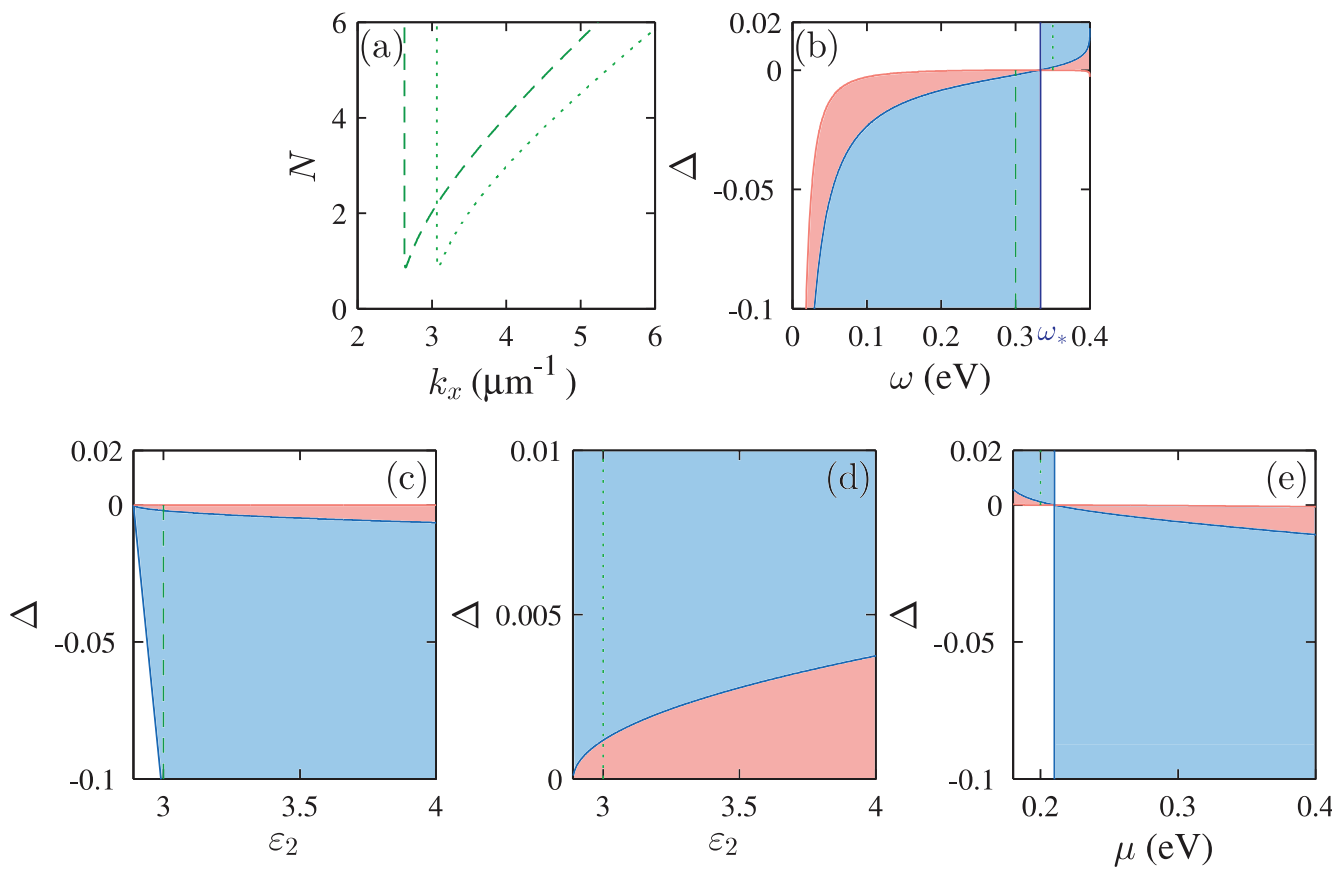

FIG. 4. (Color online) (a) Soliton norm $N$ versus wave vector $k_{x}$ for $\omega=0.3 \mathrm{eV}$ (dashed line) and $\omega=0.35 \mathrm{eV}$ (dotted line). (b)-(e) Regions of stability and instability of the nonlinear surface waves (domains shaded in blue and red, respectively) in the $(\Delta, \omega)[(\mathrm{b})],\left(\Delta, \varepsilon_{2}\right)[(\mathrm{c})$ and (d)], or $(\Delta, \mu)[(\mathrm{e})]$ planes. Other parameters are $\varepsilon_{1}=2.89, \varepsilon_{2}=3[(\mathrm{~b})$ and (e) $], \mu=0.2 \mathrm{eV}[(\mathrm{b})-(\mathrm{d})], \omega=0.3 \mathrm{eV}[(\mathrm{c})]$, or $\omega=0.35 \mathrm{eV}$ [(d) and (e)]. Dashed and dotted vertical lines in (b)-(e) correspond to the respective curves in (a). 


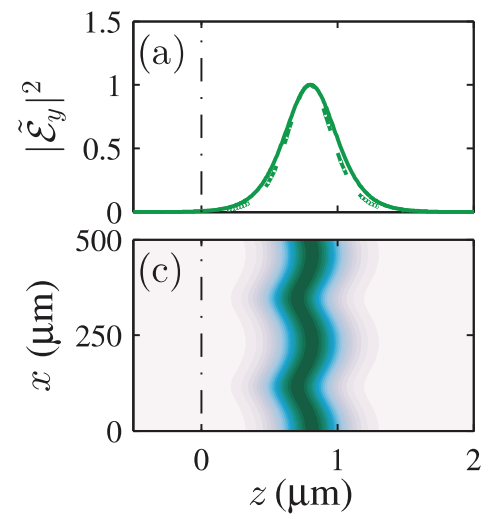

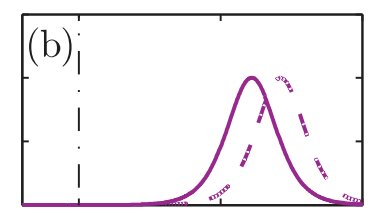
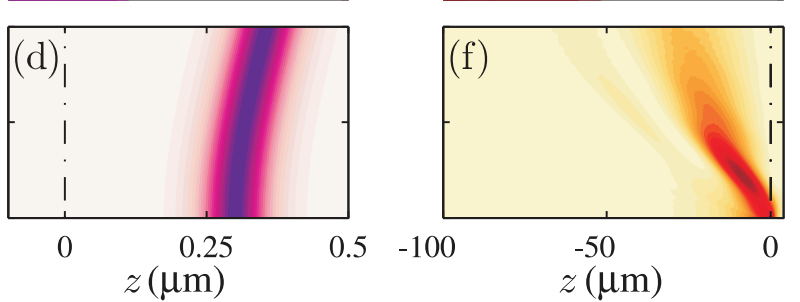

FIG. 5. (Color online) Evolution of the spatial profile of the nonlinear surface polariton as it propagates along $x$. In (a), (b), and (e) solid and dashed lines correspond to the initial (at $x=0 \mu \mathrm{m}$ ) and final (at $x=500 \mu \mathrm{m}$ ) wave profiles, respectively. Parameters of (a), (c) and (b), (d) correspond to points A in Fig. 2 and $\mathrm{B}^{\prime}$ in Fig. 3 of the paper, respectively, while those of (e) and (f) are $\Delta=-3.8 \times 10^{-4}, \omega=0.3 \mathrm{eV}, \mu=0.2$ $\mathrm{eV}$. Shown is the beam intensity $\left|\tilde{\mathcal{E}}_{y}(x, z)\right|^{2}$ normalized to unity. The dash-dotted line marks the interface between the linear and nonlinear dielectrics.

in a wide range, from almost zero up to the velocity of light in the linear dielectric $c / \sqrt{\varepsilon_{2}}$.

\section{STABILITY OF NONLINEAR WAVES}

When dealing with the solutions of a given nonlinear problem, the central question concerns the stability of these solutions. To address this issue, we introduce the norm $N$ as a dimensionless integral:

$$
N=\frac{\omega}{c} \int \mathcal{E}_{y}^{2}(z) d z=\frac{c}{\omega} \frac{\left(p_{1}+p_{2}\right)^{2}-\left(\omega \mu_{0} \sigma^{\prime \prime}\right)^{2}}{2 p_{2}} .
$$

In the case $\varepsilon_{1}<\varepsilon_{2}$ (i.e., $\Delta_{0}<0$ ), the norm for a fixed $\omega$ has a minimum [see Fig. 4(a)] at the critical wave vector,

$$
\begin{aligned}
k_{x_{\text {crit }}}^{2}= & \frac{\omega^{2}}{c^{2}}\left(\varepsilon_{2}-\frac{\Delta_{0}+\left(c \mu_{0} \sigma^{\prime \prime}\right)^{2}}{8\left(c \mu_{0} \sigma^{\prime \prime}\right)^{2}}\left\{-3 \Delta_{0}+\left(c \mu_{0} \sigma^{\prime \prime}\right)^{2}\right.\right. \\
& \left.-\sqrt{\left[\Delta_{0}+\left(c \mu_{0} \sigma^{\prime \prime}\right)^{2}\right]\left[9 \Delta_{0}+\left(c \mu_{0} \sigma^{\prime \prime}\right)^{2}\right]}\right) .
\end{aligned}
$$

Moreover, if $\left(c \mu_{0} \sigma^{\prime \prime}\right)^{2} \ll \varepsilon_{2}-\varepsilon_{1}$, the expression for the critical wave vector (15) reduces to

$$
k_{x_{\text {crit }}}^{2} \approx\left(\frac{\omega}{c}\right)^{2}\left[\varepsilon_{2}-\frac{\Delta_{0}+\left(c \mu_{0} \sigma^{\prime \prime}\right)^{2}}{3}\right] .
$$

In the stability analysis, the critical wave vector $k_{x_{\text {crit }}}$ plays an important role. It determines the boundary in the domain of parameters $\Delta$ and $\omega$ corresponding to stable and unstable modes. In other words, nonlinear polaritons with $k_{x}<k_{x_{\text {crit }}}$ (for which $\partial N / \partial k_{x}<0$ ) are unstable, ${ }^{16}$ while in the opposite case of $k_{x}>k_{x_{\text {crit }}}\left(\partial N / \partial k_{x}>0\right)$ the nonlinear surface wave is stable (according to the conventional Vakhitov-Kolokolov criterion for the soliton stability ${ }^{17}$ ). We point out that the presence of graphene (with $\sigma^{\prime \prime} \neq 0$ ) results in lowering the critical wave vector value (16), in comparison with the case without graphene (considered in Ref. 16).

The stable and unstable domains are depicted in Fig. 4(b). We see that for $\omega<\omega_{*}$ (when $\sigma^{\prime \prime}>0$ ) the nonlinear modes are unstable in the vicinity of the domain boundary, determined by Eq. $(11)$, whereas for $\omega>\omega_{*}\left(\right.$ when $\left.\sigma^{\prime \prime}<0\right)$ the unstable re- gion includes the full domain (12) as well as part of domain (13) in the vicinity of its boundary. In other words, all the nonlinear surface polaritons with $s=1$ are unstable. Fortunately, this case is not interesting since it corresponds to weakly confined nonlinear TE waves. However, both polaritons depicted in Fig. 2(d) $\left(\varepsilon_{1}<\varepsilon_{2}\right.$ and $\left.\sigma^{\prime \prime}>0\right)$ are stable and this has been confirmed by numerical integration of Eq. (1) in the paper [see Figs. 5(a) and 5(c)]. If a linear dielectric with a higher value of $\varepsilon_{2}$ is used, the widths of both [see Figs. 4(c) and 4(d)] of the above-mentioned unstable domains become larger. At the same time, in the case of fixed values of $\omega, \Delta$, and $\varepsilon_{1,2}$ the width of the unstable domain also can be varied by varying the chemical potential of graphene $\mu$ (e.g., by means of electrostatic gating). Thus, if one starts from the situation where $\omega=2 \mu$ [left side of Fig. 4(e)] and, hence, $\sigma^{\prime \prime}(\omega)<0$, the increase of the chemical potential results in a decrease of the unstable domain width, which shrinks at a particular value of $\mu_{*}$, where $\sigma^{\prime \prime}(\omega)=0$. Further growth of $\mu$ [in the region where $\sigma^{\prime \prime}(\omega)>0$ ], leads to the broadening of the unstable domain.

In the opposite case $\left(\varepsilon_{1}>\varepsilon_{2}\right.$ and $\left.\sigma^{\prime \prime}<0\right)$ the norm $N$ of the localized waves does not have a minimum and grows monotonically with the increase of $k_{x}$, i.e., we have the stability of the nonlinear polaritons in the full existence domain. Numerical integration of Eq. (1) shows [see Figs. 5(b) and 5(d)] that the nonlinear wave is not collapsing and maintains its shape. In this sense, the Vakhitov-Kolokolov criterion is not violated here. At the same time, the nonlinear wave undergoes a drift instability, ${ }^{18}$ so that in the course of propagation the center-of-mass of the spatial soliton in the nonlinear medium gradually moves away from the interface $z=0$.

An example of spatial evolution of the unstable soliton is depicted in Figs. 5(e) and 5(f). Here the main part of the power initially is concentrated in the half-space $z<0$, occupied by the linear dielectric [Fig. 5(e)]. During the spatial evolution the soliton center starts moving in the negative direction of $z$ axis [Fig. 5(f)], and its initial shape is destroyed.

\section{CONCLUSIONS}

We have studied, both analytically and numerically, the propagation of electromagnetic waves along the surface of a 
nonlinear dielectric medium covered by a layer of graphene. We have demonstrated that the presence of a single graphene layer leads to the existence and stabilization of nonlinear surface modes with the maximum amplitude located either at the interface or inside the nonlinear dielectric medium. This is a new effect, since no TE-polarized surface modes are known to exist at the interface of two linear dielectric media without graphene, and the modes are substantially different in the nonlinear case when the graphene layer is absent. We have studied the stability of the graphene-supported nonlinear modes and found the regions of stability; we have also discussed the scenarios of the instability-induced dynamics. Our results can be extended to the case of a single layer of graphene cladded by two nonlinear dielectrics, where we expect to have stronger localization of the mode with a maximum at the graphene layer, and also a new type of nonlinear surface modes with two maxima.

\section{ACKNOWLEDGMENTS}

Y.V.B. thanks the Nonlinear Physics Center at the Australian National University for warm hospitality during his visit at the initial stage of this project. This work was partially supported by the FEDER COMPTETE Program and by the Portuguese Foundation for Science and Technology (FCT) through Grant PEst-C/FIS/UI0607/2013. We acknowledge a support from the EC under Graphene Flagship (Contract No. CNECT-ICT-604391). The authors thank I. Iorsh and I. Shadrivov for useful discussions. *bludov@ fisica.uminho.pt

${ }^{1}$ M. Kauranen and A. V. Zayats, Nat. Photon. 6, 737 (2012).

${ }^{2}$ A. N. Grigorenko, M. Polini, and K. S. Novoselov, Nat. Photon. 6, 749 (2012).

${ }^{3}$ Yu. V. Bludov, A. Ferreira, N. M. R. Peres, and M. I. Vasilevskiy, Int. J. Mod Phys B 27, 1341001 (2013); X. Luo, T. Qiu, W. Lu, and Z. Ni, Mater. Sci. Eng.: R: Rep. 74, 351 (2013).

${ }^{4}$ F. H. L. Koppens, D. E. Chang, and F. J. G. de Abajo, Nano Lett. 11, 3370 (2011); M. Jablan, H. Buljan, and M. Soljacic, Opt. Express 19, 11236 (2011); A. Y. Nikitin, F. Guinea, F. J. Garcia-Vidal, and L. Martin-Moreno, Phys. Rev. B 84, 195446 (2011).

${ }^{5} \mathrm{Z}$. Fei et al., Nature (London) 487, 82 (2012); J. Chen et al., ibid. 487, 77 (2012).

${ }^{6}$ S. A. Mikhailov and K. Ziegler, Phys. Rev. Lett. 99, 016803 (2007).

${ }^{7}$ X. Y. He, J. Tao, and B. Meng, Nanotechnology 24, 345203 (2013).

${ }^{8}$ Q. Bao, H. Zhang, B. Wang, Z. Ni, C. H. Y. X. Lim, Y. Wang, D. Y. Tang, and K. P. Loh, Nat. Photon. 5, 411 (2011).
${ }^{9}$ Yu. V. Bludov, N. M. R. Peres, and M. I. Vasilevskiy, J. Opt. 15, 114004 (2013).

${ }^{10}$ P. I. Buslaev, I. V. Iorsh, I. V. Shadrivov, P. A. Belov, and Yu. S. Kivshar, JETP Lett. 97, 535 (2013).

${ }^{11}$ Except the situation, where substrate is a magnetic material, ${ }^{7}$ characterized by an extremely high magnetic permeability $\sim 5000$, which nowadays seems to be hardly achievable experimentally.

${ }^{12}$ M. L. Nesterov, J. Bravo-Abad, A. Yu. Nikitin, F. J. García-Vidal, and L. Martin-Moreno, Laser Photon. Rev. 7, L7 (2013).

${ }^{13}$ R. W. Boyd, Nonlinear Optics (Academic, San Diego, CA, 1992).

${ }^{14}$ W. J. Tomlinson, Opt. Lett. 5, 323 (1980).

${ }^{15}$ L. Wang, W. Cai, X. Zhang, and J. Xu, Opt. Lett. 37, 2730 (2012).

${ }^{16}$ N. N. Akhmediev, V. I. Korneyev, and Yu. V. Kuz'menko, Zh. Eksp. Teor. Fiz. 88, 107 (1985) [Sov. Phys. JETP 61, 62 (1985)].

${ }^{17}$ N. G. Vakhitov and A. A. Kolokolov, Radiophys. Quantum Electron. 16, 783 (1973).

${ }^{18}$ Y. Sivan, Ph.D. thesis, Tel Aviv University, 2009. 